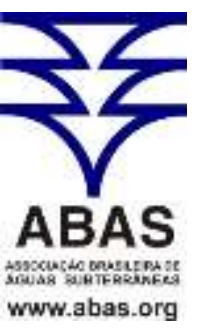

\title{
SIMULAÇÃO DE FLUXO E TRANSPORTE DE ÍONS DE VINHAÇA ATRAVÉS DE VERTENTE DA FORMAÇÃO RIO CLARO
}

\author{
SIMULATION OF WATER FLOW AND ION TRANSPORT FROM VINASSE IN \\ A TRANSECT OF THE RIO CLARO FORMATION
}

\author{
Miguel Angel Alfaro Soto ${ }^{1}$; Juliana Broggio Basso ${ }^{2}$; Chang Hung Kiang ${ }^{3}$; \\ Martinus Th. van Genuchten ${ }^{4}$
}

Artigo recebido em: 10/02/2015 e aceito para publicação em: 26/06/2015.

DOI: http://dx.doi.org/10.14295/ras.v29i2.28239

\begin{abstract}
Vinasse is increasingly used in agricultural operations as a possible source of nutrients and organic matter. This paper presents results of simulations of groundwater flow and ion transport from the hypothetical infiltration of vinasse from fertigation of sugar cane. The study was applied to a two-dimensional vertical transect in Ajapí (Rio Claro - SP). The main objective was to assess potential contamination of groundwater by vinasse constituents, and their transport to a stream close to sources of infiltration. Numerical simulations were carried out with the HYDRUS 2D/3D software package using representative data of the physical soil and hydrogeological properties of the area. Calculations assumed a steady-state flow regime in a layered but isotropic porous medium representing the Rio Claro formation of sediments. Results show that for the hypothetical scenarios considered, ions derived from vinasse may have a high potential of contaminating both groundwater and nearby streams. This serves as a warning about the vulnerability of areas with similar scenarios involving the extensive use of vinasse through fertigation.
\end{abstract}

Keywords: Contaminant transport. Vinasse. Fertigation. Numerical model. HYDRUS 2/3D.

Resumo: A vinhaça é cada vez mais usada nas operações agrícolas como uma possível fonte de nutrientes e matéria orgânica. Neste artigo, são apresentados os resultados de uma simulação de fluxo subterrâneo da água e de transporte de íons, a partir da hipotética infiltração de vinhaça decorrente da fertirrigação na cultura de cana de açúcar. O estudo é aplicado a um corte bidimensional de solo de Ajapí (Rio Claro - SP). As simulações tiveram com a finalidade de avaliar a potencial contaminação das águas subterrâneas e de um córrego localizado próximo às fontes de infiltração. Foi realizada uma modelagem numérica empregando o software HYDRUS 2D/3D, usando dados representativos das propriedades físicas e hidrogeológicas do meio no local. Os cálculos consideram os fluxos da água e da vinhaça em regime permanente, em um meio poroso homogêneo e isotrópico representando os sedimentos da Formação Rio Claro. Os resultados obtidos apontaram que para os cenários hipotéticos, aqui apresentados, íons provenientes da vinhaça, em determinadas concentrações, podem apresentar alto potencial de contaminação tanto de águas subterrâneas como superficiais. Os resultados servem como alerta para a vulnerabilidade de áreas com cenários similares, quais sejam, de intensa fertirrigação com vinhaça.

Palavras-chave: Transporte de contaminantes. Vinhaça. Fertirrigação. Modelo numérico. HYDRUS 2/3D.

\section{INTRODUÇÃO}

O Brasil é o maior produtor de açúcar e etanol no mundo. A área cultivada que será colhida e destinada à atividade sucroalcooleira na safra 2014/2015 será de aproximadamente 9.004,5 mil hectares, distribuídas em todos os estados produtores, sendo São Paulo o maior produtor, com 52\% de área plantada (CONAB, 2014). A produção sucroalcooleira é responsável pela geração da vinhaça, que é seu principal resíduo, usado na complemen-

\footnotetext{
${ }^{1}$ LEBAC - Laboratório de Estudo de Bacias, Departamento de Geologia Aplicada, UNESP - Campus de Rio Claro (alfaro@rc.unesp.br)

${ }^{2}$ LEBAC - Laboratório de Estudo de Bacias, Departamento de Geologia Aplicada, UNESP - Campus de Rio Claro(julibb@rc.unesp.br)

${ }^{3}$ LEBAC - Laboratório de Estudo de Bacias, Departamento de Geologia Aplicada, UNESP - Campus de Rio Claro (chang@rc.unesp.br)

4 COPPE/LTCC, Departamento de Engenharia Mecânica, UFRJ - Universidade Federal de Rio de Janeiro (rvangenuchten@hotmail.com)
} 
tação da adubação do solo em algumas culturas e inclusive na própria cana de açúcar. No entanto, seu uso em quantidades relativamente grandes pode ser questionável devido a problemas ambientais produzidos pela fertirrigação, quando se leva em consideração que a vinhaça é rica em matéria orgânica e potássio, com significativos teores de cálcio, magnésio, nitratos e enxofre.

Os impactos da vinhaça no ambiente, com a aplicação excessiva (superiores à estabelecida pela CETESB, 2006) e contínua desse efluente com alta carga orgânica (DBO/DQO), baixo $\mathrm{pH}$, elevada corrosividade, e presença de cátions e metais em solução, podem advir da infiltração que causa contaminação de águas subterrâneas e salinização de aquíferos. Consequentemente, o conhecimento da distribuição da água e dos solutos no meio poroso é de grande importância na avaliação de problemas deste tipo. Questões como essas têm sido geralmente abordadas mediante trabalhos conduzidos em laboratório.

Brito et al. (2007) avaliaram a qualidade do lixiviado de solos (Nitossolo Háplico, Argissolo amarelo e Espodossolo Cárbico) que receberam diferentes doses de vinhaça em diferentes tempos de incubação. Eles utilizaram testes em colunas com solos de 20 $\mathrm{cm}$ de diâmetro e $110 \mathrm{~cm}$ de altura. As análises para quantificação de $\mathrm{K}, \mathrm{Ca}, \mathrm{Mg}$ e $\mathrm{Na}$ nos efluentes coletados mostraram que os valores dos cátions do lixiviado foram significativamente reduzidos em relação aos da vinhaça in natura, indicando o elevado poder dos solos de retenção dos vários constituintes da vinhaça.

Uyeda et al. (2013) utilizaram colunas com diferentes tipos de solos (Latossolo vermelho escuro, Latossolo roxo, Nitossolo eutrófico vermelho) como permeâmetros a carga constante para percolação de quatro doses de vinhaça, com a finalidade de testar a hipótese de redução da condutividade hidráulica devido aos efeitos dispersivos das partículas de argila pela presença principalmente do $\mathrm{K}$ e $\mathrm{Na}$. Os resultados confirmaram a redução da condutividade hidráulica para o Latossolo vermelho escuro apenas para apli- cações superiores a $300 \mathrm{~m}^{3} / \mathrm{ha}$, bem como aumentos de $\mathrm{K}$ e $\mathrm{Ca}$, e da capacidade de troca de cátions.

Outros trabalhos como o de Da Silva et al. (2012) utilizaram a modelagem numérica com a finalidade de obter os parâmetros de transporte do $\mathrm{Na}$ e $\mathrm{K}$ decorrentes da vinhaça, velocidade de escoamento da água, fator de retardamento, dispersividade e coeficiente de dispersão, a partir de testes em colunas em um Nitossolo Vermelho Eutrófico. Após simulação numérica, os resultados evidenciaram os efeitos de adsorção e deslocamento de $\mathrm{Na}$ e principalmente do K presentes na vinhaça.

Na natureza, o transporte dos solutos depende de muitos fatores físicos e químicos, dentre eles as propriedades do meio, do fluido percolante e as condições ambientais. No entanto, a dependência entre os parâmetros de transporte envolvidos tornam a modelagem uma tarefa complexa, recorrendo ao emprego da simulação numérica que utiliza intervalos discretos de espaço e tempo. Modelos de simulação numérica são atualmente muito utilizados para auxiliar no entendimento dos complexos processos de deslocamento de solutos em perfis de solos saturados e não saturados, sendo importantes ferramentas para o gerenciamento de tomadas de decisão e redução de impactos ao meio ambiente. Embora de grande utilidade, a literatura especializada não tem se ocupado de trabalhos que utilizem modelagem numérica com vistas à preservação de águas subterrâneas e superficiais na região de São Paulo.

Nesse contexto, este trabalho visa elucidar o potencial grau de contaminação por vinhaça das águas subterrâneas e superficiais em sedimentos da Formação Rio Claro, a partir da fertirrigação de cultivo de cana de açúcar. Cabe ressaltar que essa unidade constitui um importante aquífero utilizado como fonte de abastecimento local de água, em grande parte para consumo humano.

\section{2 ÁREA DE ESTUDO}

A área de estudo se localiza no distrito industrial de Ajapi, município de Rio Cla- 
ro (São Paulo). Sua escolha se justifica por se tratar de uma região, cujo solo é tipicamente utilizado para cultivo de cana de açúcar. A Figura 1 mostra um mapa de localização da área de estudo. A área se encontra cercada por áreas industriais, de cultivo de cana de açúcar e pelo córrego Antônio Chiaradia. O local constitui uma vertente onde a porção superior pertence à Formação Rio Claro. Essa unidade é formada por depósitos fluviais de idade Cenozóica, pertencentes à Bacia Sedimentar do Paraná. A unidade é constituída predominantemente por arenitos, em geral de granulometria fina a média, cujo solo é classificado como latossolo vermelhoamarelo (LVA, 1984).

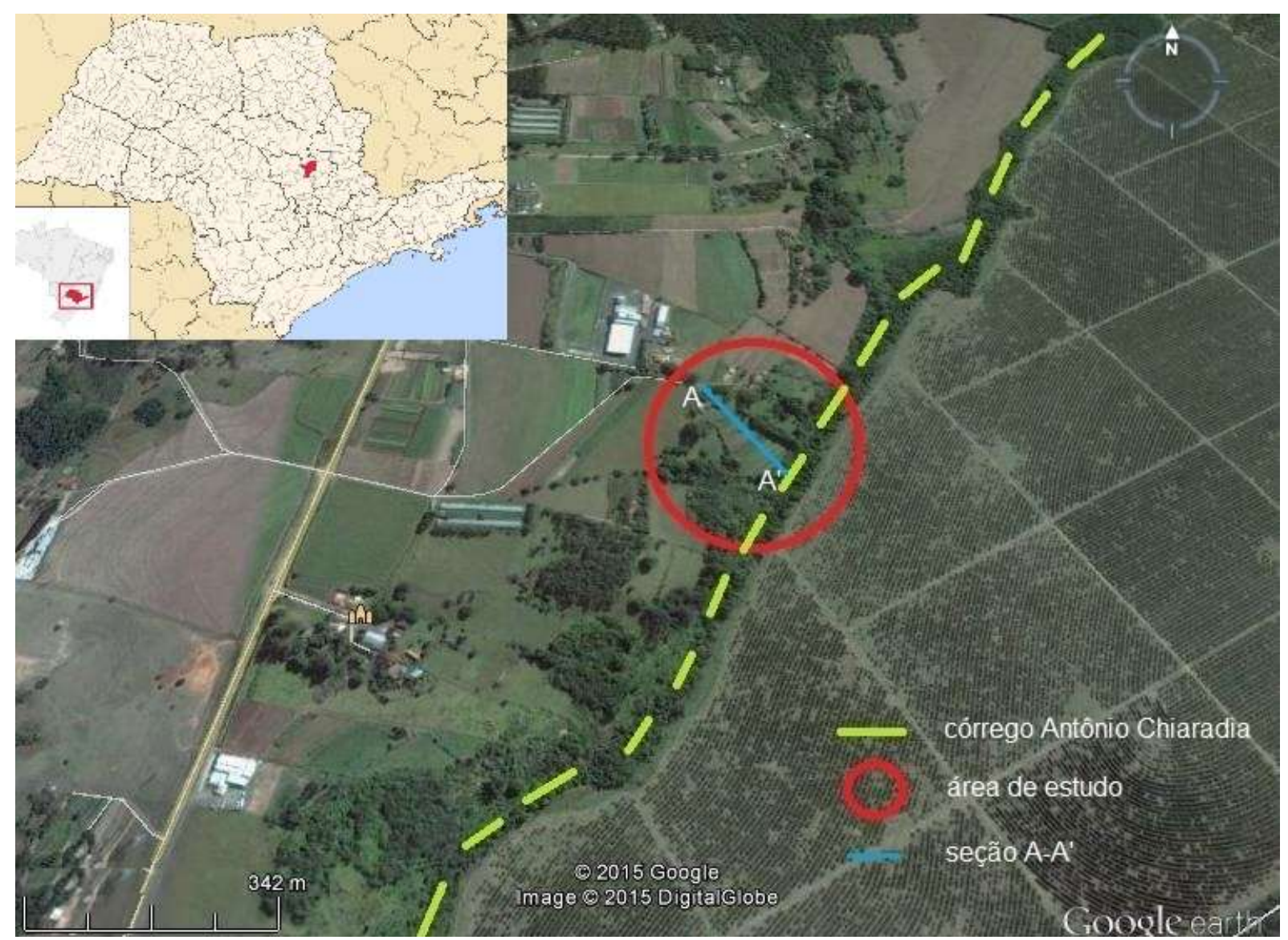

Figura 1 - Localização da área de estudo (Google Earth, 2014). Seção AA’ é apresentada na Figura 2

Figure 1- Location of the study area (Google Earth, 2014). Section AA' is shown in Figure 2

Apesar de apresentar extensão territorial restrita (em relação a outras unidades da bacia), a Formação Rio Claro constitui um importante aquífero livre sobre o qual a atividade canavieira tem apresentado grande crescimento, com aplicação frequente de grande volume de vinhaça no solo (fertirrigação). O substrato dessa unidade na área é formado pela Formação Corumbataí que, segundo IPT (1981), é caracterizada por siltitos, arenitos finos a médios, silto-argilosos, de origem marinha, em parte concrecionados por calcário e sílex.

\section{MÉTODOS TEÓRICOS E EXPERI- MENTAIS}

Neste item são mostrados aspectos do modelo conceitual da área de estudo que reúne a informação referente à fonte de contaminação, rotas de exposição e os receptores, bem como os dados necessários (parâmetros físicos e de contaminantes) para calcular as concentrações finais, isto é, nos pontos de interesse, a partir de modelos numéricos contidos no software HYDRUS 2D/3D (SIMUNEK et al.,1999). 


\subsection{Modelo conceitual da área de estudo}

Estipulou-se, hipoteticamente, que uma fonte de contaminação proveniente da fertirrigação com vinhaça inicia-se a partir da superfície do solo, onde a concentração inicial infiltra-se verticalmente ao longo da zona não saturada até atingir o aquífero granular (zona saturada). Nessa zona, a vinhaça passa por um processo natural de diluição, deslocando-se em sentido horizontal com a água subterrânea até atingir o córrego Antônio Chiaradia.

Para simular o transporte dos solutos na água subterrânea até a água superficial, foi utilizado o software HYDRUS 2D/3D (SI-
MUNEK et al.,1999). Para tanto, estabeleceu-se um modelo conceitual considerando a localização, as dimensões geométricas, as características físicas e hidráulicas do meio, bem como dos contaminantes, que serviram como parâmetros de entrada para a simulação. A Figura 2 mostra um esquema do perfil de solo com as características geométricas, fontes de vinhaça, rotas de exposição e potenciais receptores dos contaminantes na área de estudo. A seção avaliada apresenta aproximadamente 700 metros de comprimento e 50 metros de altura. Hipoteticamente, o transporte se iniciou na superfície da Formação Rio Claro, sobreposta à Formação Corumbataí.

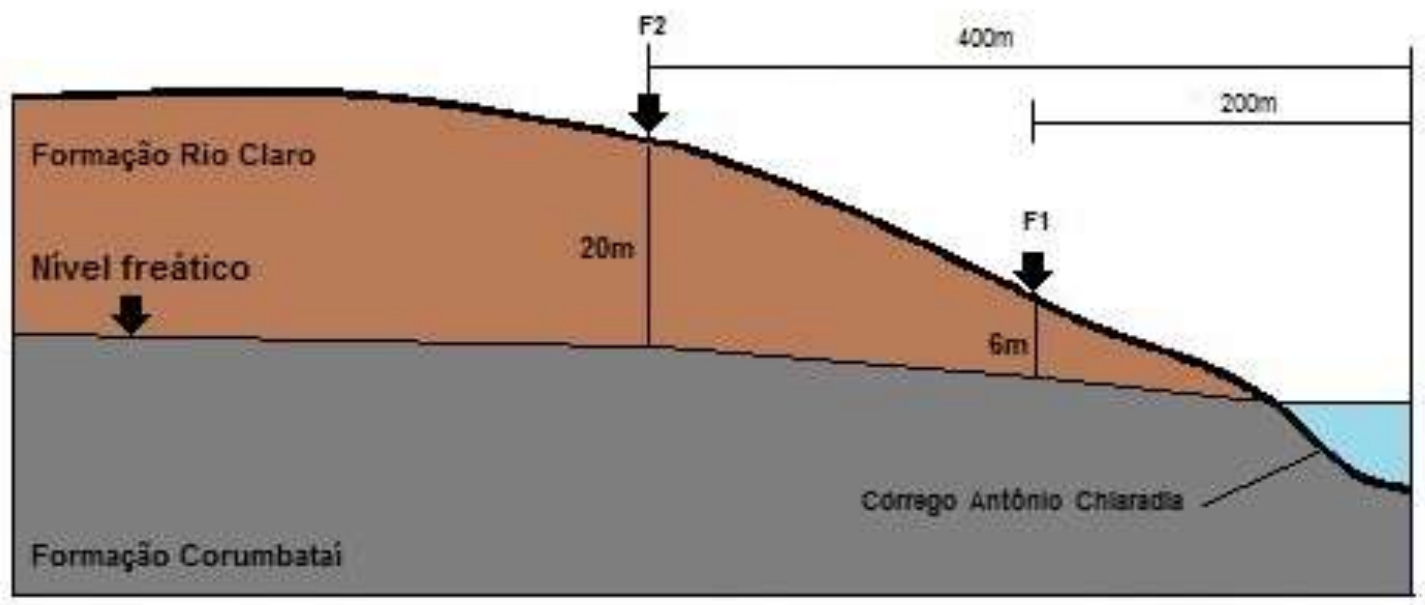

Figura 2 - Seção A-A' características geométricas, fontes de contaminação (F1 a 200 metros do córrego e F2 a 400 metros do córrego), rotas de exposição e potenciais receptores

Figure 2 - Section A-A 'geometric characteristics, sources of contamination (F1- 200 meters from the stream and F2- 400 meters from the stream), exposure media and potential receptors

Com o intuito de simplificar o problema e devido a não se contar com dados experimentais suficientes, assumiu-se a isotropicidade e homogeneidade dos solos de cada formação. De forma arbitrária, foram estabelecidas duas fontes de vinhaça, com 80 metros de comprimento cada, com as fontes localizadas a 200 metros do córrego $(\mathrm{F} 1)$ e a 400 metros do córrego (F2). Os cálculos foram focados na relação entre distância, tempo de chegada e concentração final no córrego Antônio Chiaradia.

\subsection{Caracterização física e hidráulica}

Amostras indeformadas das forma- ções Rio Claro e Corumbataí foram utilizadas para ensaios de caracterização física (granulometria, massas específicas do solo e dos grãos), além desses, foram realizados testes para determinação da curva de retenção de água no solo pelo método do papel filtro (Marinho, 1994) e para determinação da condutividade hidráulica saturada com o permeâmetro Guelph (REYNOLDS e ELRICK, 1995).

Quanto ao ajuste de curvas de retenção foi necessário o emprego do modelo de Durner (1994), que é mais apropriado para solos com distribuições bimodais de poros, tal como será comentado mais adiante. O modelo é descrito por: 


$$
S_{e}=\sum_{i=1}^{k} w_{i}\left(1+\left|\alpha_{i} h\right|^{n_{i}}\right)^{-m_{i}}
$$

onde $S_{e}$ representa a saturação eficaz, $h$ é a carga de pressão, $k$ representa o número de subsistemas que formam a distribuição total de poros (2 em nosso estudo), e $w_{i}$ são os fatores designados para os pesos das subcurvas que variam entre 0 e 1 , isto é $\Sigma w_{i}=1$. Da mesma forma que no modelo unimodal de van Genuchten (1980), os parâmetros das subcurvas (i.e., $\alpha_{i}$, e $n_{i}$ ) estão sujeitos à condicionantes: $\alpha_{i}>0$ e $n_{i}>1$, enquanto que $m_{i}=1-1 / n_{i}$.

Também utilizou-se a função condutividade hidráulica, obtida a partir da curva de retenção e da condutividade hidráulica saturada. No entanto, devido às características de retenção da água, foi necessário adotar também a versão bimodal do modelo de van Genuchten (Durner, 1994), dado por:

$$
K\left(S_{e}\right)=K_{s}\left(\sum_{i=1}^{k} w_{i} S_{e_{i}}\right)^{0,5} \frac{\left(\sum_{i=1}^{k} w_{i} \alpha_{i}\left[1-\left(1-S_{e_{i}}^{1 / m}\right)^{m}\right]\right)^{2}}{\left(\sum_{i=1}^{k} w_{i} \alpha_{i}\right)^{2}}
$$

onde $K\left(S_{e}\right)$ representa a função condutividade hidráulica e $K_{s}$ é a condutividade hidráulica do solo saturado.

\subsection{Transporte de solutos}

O software HYDRUS 2D/3D (Simunek et al.,1999) considera o fluxo de água utilizando a equação geral de Richards e a equação de advecção-dispersão para o transporte do soluto em um meio variavelmente saturado. Para um meio isotrópico bidimensional, a equação de Richards é dada por:

$$
\frac{\partial \theta(h)}{\partial t}=\frac{\partial}{\partial x_{i}}\left(K_{i j} \frac{\partial h}{\partial x_{j}}-K_{i j}\right)-S(h), \quad(i, j=1,2)
$$

onde $\theta$ é a umidade volumétrica, $x_{i}(i=1,2)$ são as coordenadas espaciais, $t$ o tempo, $K_{i j}$ os componentes do tensor de condutividade hidráulica, e $S(h)$ o termo fonte (ou sumidou- ro) de água. Uma vez que neste estudo o meio foi considerado isotrópico, o tensor de condutividade é diagonal, onde $K_{x x}$ e $K_{y y}$ tornam-se iguais à condutividade hidráulica não saturada, $K(h)$ ou $K(\theta)$.

O transporte de soluto pode ser descrito usando a equação de advecçãodispersão, dada por:

$$
\frac{\partial(\theta R C)}{\partial t}=\frac{\partial}{\partial x_{i}}\left(\theta D_{i j} \frac{\partial C}{\partial x_{j}}\right)-\frac{\partial q C}{\partial x_{i}}, \quad(i, j=1,2)
$$

onde $C$ representa a concentração em solução, $q$ o fluxo de água, e $D_{i j}$ o tensor de dispersão que pode ser descrito em termos de dispersividade longitudinal $\left(D_{L}\right)$, a dispersividade transversal $\left(D_{T}\right)$, e o coeficiente de difusão molecular $\left(D_{w}\right)$. O termo $R$ na equação (4) refere-se ao fator de retardamento considerando adsorção em equilíbrio.

Cabe ressaltar que, os parâmetros de dispersividade $\left(D_{L}\right.$ e $\left.D_{T}\right)$ foram estimados segundo recomendações da ASTM (1995). Adicionalmente, o coeficiente de difusão é modificado pelo fator de tortuosidade $(\tau)$, utilizando-se a relação de Millington e Quirk (1961).

\section{RESULTADOS}

Neste item são apresentados os resultados da caracterização física, hidráulica, bem como da simulação numérica obtidos para o presente estudo.

\subsection{Caracterização física e hidráulica}

As Figuras 3 e 4 mostram as curvas de retenção de água obtidas para os solos das formações Rio Claro e Corumbataí, respectivamente. Verifica-se que, para as duas unidades, as curvas de retenção são típicas de solos com distribuição bimodal de poros. Consequentemente, o ajuste da curva aos dados experimentais em ambos os solos foi realizado utilizando a Equação (1) de Durner (1994) que permitiu um adequado ajustamento aos dados experimentais. Segundo Alfaro Soto e Chang (2008), este tipo de curva de 
retenção é comum em solos do estado de São Paulo (entre outros estados) e decorre da es- truturação de solos de características lateríticas.

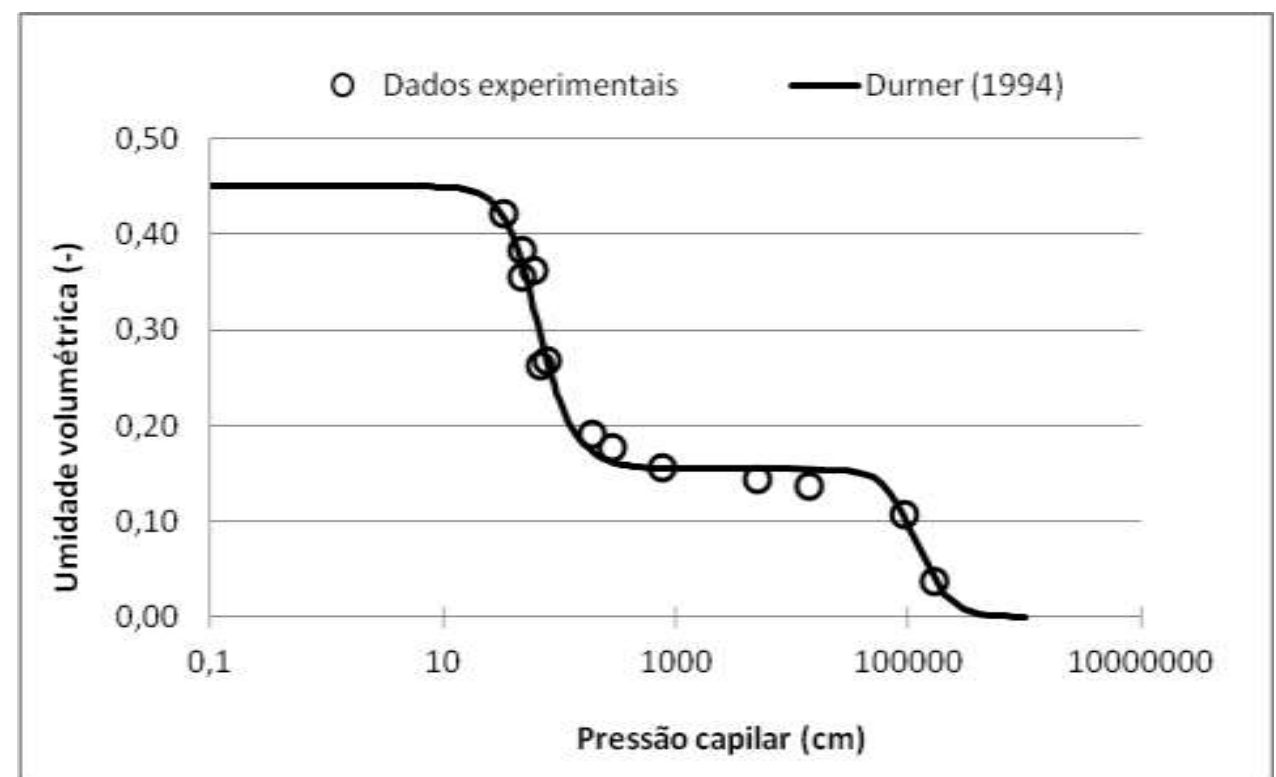

Figura 3 - Curva de pressão capilar da Formação Rio Claro

Figure 3 - Capillary pressure curve of the Rio Claro Formation

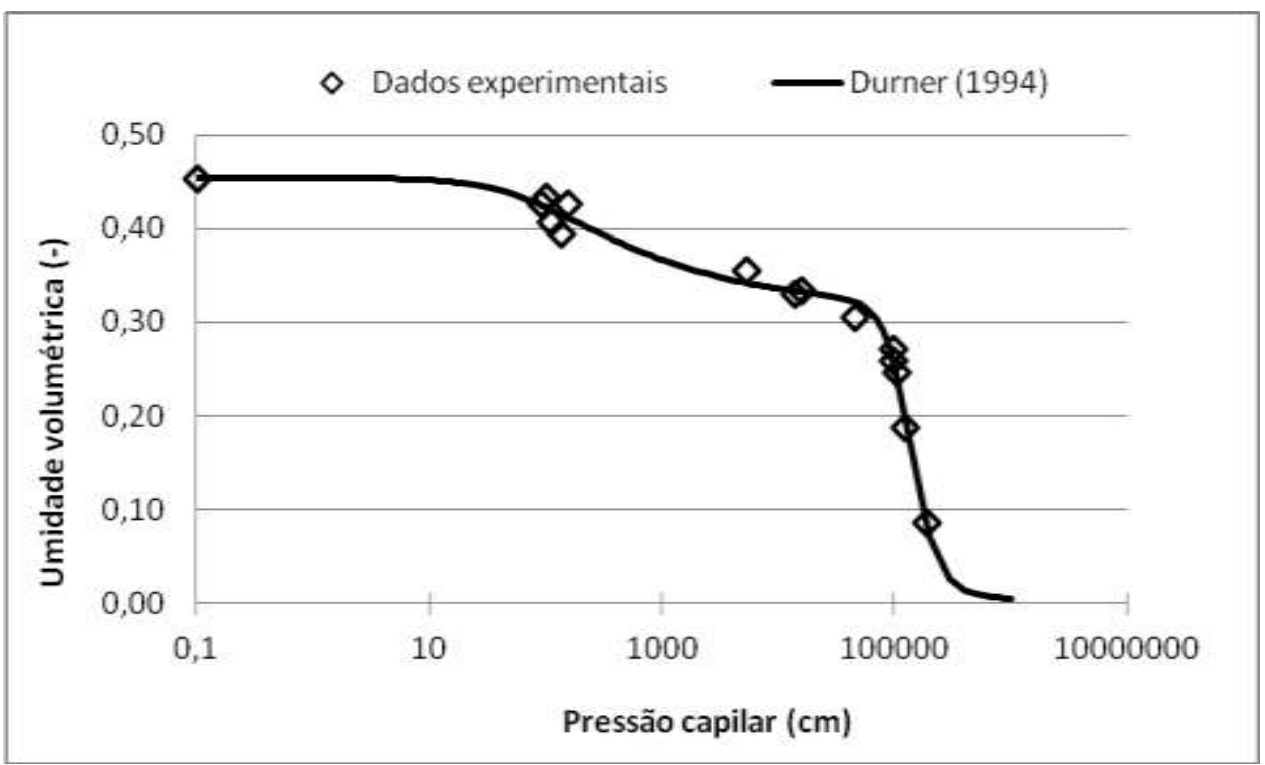

Figura 4 - Curva de pressão capilar da Formação Corumbataí

Figure 4 - Capillary pressure curve of the Corumbataí Formation

Adicionalmente, a partir dos dados do ajuste da curva de retenção e testes de condutividade hidráulica saturada foi possível a determinação da função condutividade hidráulica não saturada mediante a Equação
(2). A Figura 5 mostra as curvas das funções $K(h)$ para os dois tipos de solos, evidenciando a peculiaridade da função condutividade hidráulica. 


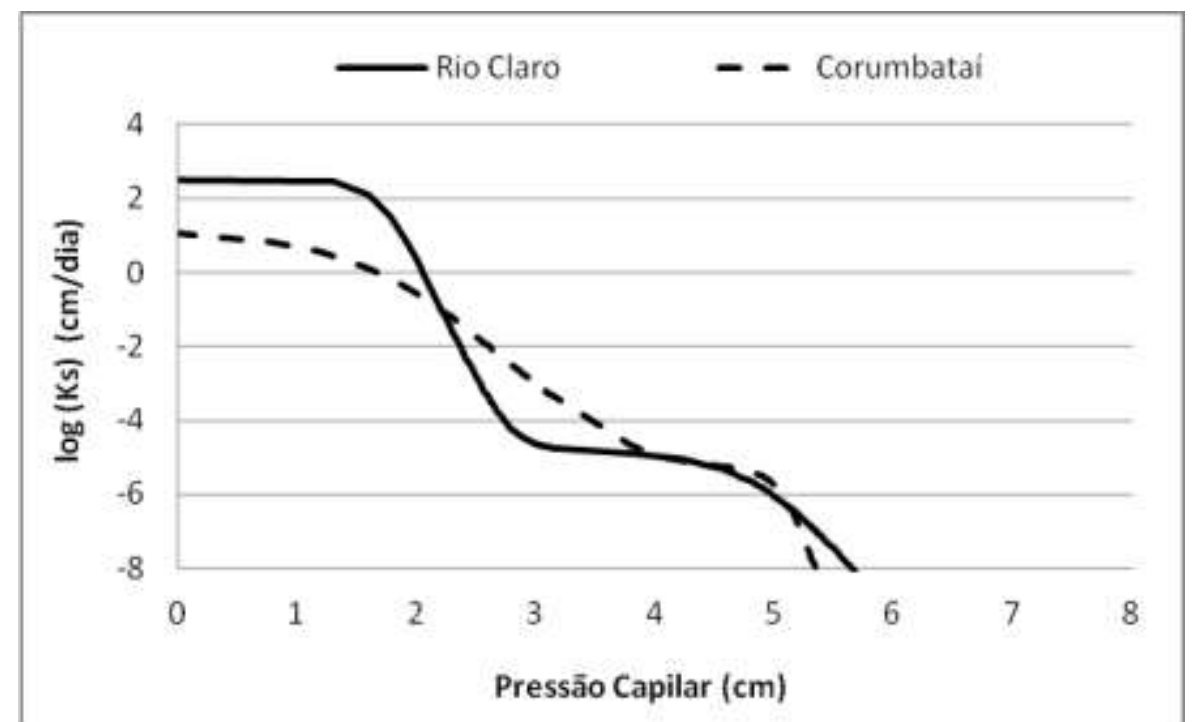

Figura 5- Função condutividade hidráulica (não saturada) dos solos das formações Rio Claro e Corumbataí

Figure 5 - Hydraulic conductivity function (unsaturated) of Rio Claro and Corumbataí formations

Como já apontado por Alfaro Soto e Chang (2013) a importância da bimodalidade em solos reside na significativa diferença de condutividade hidráulica não saturada para um mesmo valor de pressão capilar caso seja considerado um modelo unimodal. Consequentemente, este fato sugere que é necessário o uso da função bimodal, caso contrário, podem ser também obtidos discrepantes concentrações finais dos solutos nos pontos de exposição (água subterrânea e córrego). A Tabela 1 resume os parâmetros físicos e hidráulicos obtidos para os solos das formações Rio Claro e Corumbataí, utilizados na simulação.

Tabela 1 Características físicas e hidráulicas representativas dos solos das Formações Rio Claro e Corumbataí Table 1 - Physical and hydraulic characteristics of soils from Rio Claro and Corumbataí formations

\begin{tabular}{lccc}
\hline Parâmetro & Unidade & $\begin{array}{c}\text { Formação } \\
\text { Rio Claro }\end{array}$ & $\begin{array}{c}\text { Formação } \\
\text { Corumbataí }\end{array}$ \\
\hline Textura & - & Areia argilosa & $\begin{array}{c}\text { Silte arenoso } \\
\text { Massa específica seca, } \rho d\end{array}$ \\
Massa específica dos sólidos, $\rho_{s}$ & $\mathrm{~g} / \mathrm{cm} 3$ & 1,37 & 1,39 \\
Umidade volumétrica saturada, $\theta_{s}$ & $\mathrm{~g} / \mathrm{cm}^{3}$ & 2,60 & 2,76 \\
Umidade volumétrica residual, $\theta_{r}$ & $\mathrm{~cm}^{3} / \mathrm{cm}^{3}$ & 0,45 & 0,454 \\
Parâmetro da subcurva 1, $\alpha_{1}$ & $\mathrm{~cm}^{3} / \mathrm{cm}^{3}$ & 0,00 & 0,00 \\
Parâmetro da subcurva 1, $n_{1}$ & $\mathrm{~cm}^{-1}$ & 0,0178 & 0,0136 \\
Peso da subcurva 1, $\mathrm{w}_{1}$ & $(-)$ & 3,270 & 1,360 \\
Parâmetro da subcurva 2, $\alpha_{2}$ & $(-)$ & 0,656 & 0,314 \\
Parâmetro da subcurva 2, $n_{2}$ & $\mathrm{~cm}^{-1}$ & $1,0 \mathrm{E}-5$ & $8,0 \mathrm{E}-06$ \\
Condutividade hidráulica saturada, $K_{s}$ & $(-)$ & 1,680 & 4,220 \\
\hline
\end{tabular}

Como sabido, os impactos da vinhaça em aquíferos são decorrentes de suas propriedades e composição (DBO/DQO, pH, corrosividade, cátions, metais, etc). Porém, para esta simulação de transporte de solutos foi escolhido um tipo arbitrário de íon, não reativo com o meio, cuja concentração foi assumida como igual a $1 \mathrm{mmol} / \mathrm{cm}^{3}$. Desta forma, o resultado será a fração de concentração final $\left(C_{i}\right)$ em relação à inicial $\left(C_{o}\right)$, 
servindo de referência para determinação expedita do transporte de outros tipos de contaminantes ao possuírem uma relação proporcional entre eles. Para tanto, foram adotados apenas os mecanismos de advecção e dispersão hidrodinâmica, não sendo consideradas as reações de adsorção.

Devido às características intrínsecas do solo da Formação Rio Claro na área, isto é, elevada macroporosidade e fraturas, estabeleceu-se o fluxo em regime permanente ou steady state (sendo este mais conservador) com elevada taxa de recarga $(0,09 \mathrm{~cm} / \mathrm{dia})$, conforme estudos já realizados nas proximidades por Carnier e Chang (2008). A Tabela 2 compila alguns parâmetros utilizados para análise de transporte de solutos.

Tabela 2 - Parâmetros selecionados utilizados para simulação do transporte de solutos em solos das formações Rio Claro e Corumbataí

Table 2 - Parameters selected for simulation of solute transport in soils from Rio Claro and Corumbataí formations

\begin{tabular}{lccc}
\hline Parâmetro & Unidade & \multicolumn{2}{c}{ Íon da vinhaça } \\
& & Rio Claro & Corumbataí \\
\hline Distância entre foco e fonte, $x$ & $\mathrm{~m}$ & $200 \mathrm{e} 400$ & $200 \mathrm{e} 400$ \\
Dispersividade longitudinal, $D_{L}$ & $\mathrm{~m}$ & $2 \mathrm{e} 4$ & $2 \mathrm{e} 4$ \\
Dispersividade transversal, $D_{T}$ & $\mathrm{~m}$ & $0,66 \mathrm{e} 1,33$ & $0,66 \mathrm{e} 1,33$ \\
Coeficiente de difussão, $D_{w}$ & $\mathrm{~cm}^{2} /$ ano & 365 & 365 \\
Fator de retardamento, $R$ & - & - & - \\
\hline
\end{tabular}

\subsection{Simulação Numérica}

A análise de transporte de solutos contemplou o modelo bidimensional. Os dados das cargas de pressão positivas e negativas (sucções matriciais na zona não saturada) em toda a extensão do solo foram obtidos por extrapolação a partir do nível freático, onde a pressão capilar é igual a zero para o teor de umidade de saturação da Tabela 1. A Figura 6 mostra os resultados da distribuição das pressões para a condição steady state.

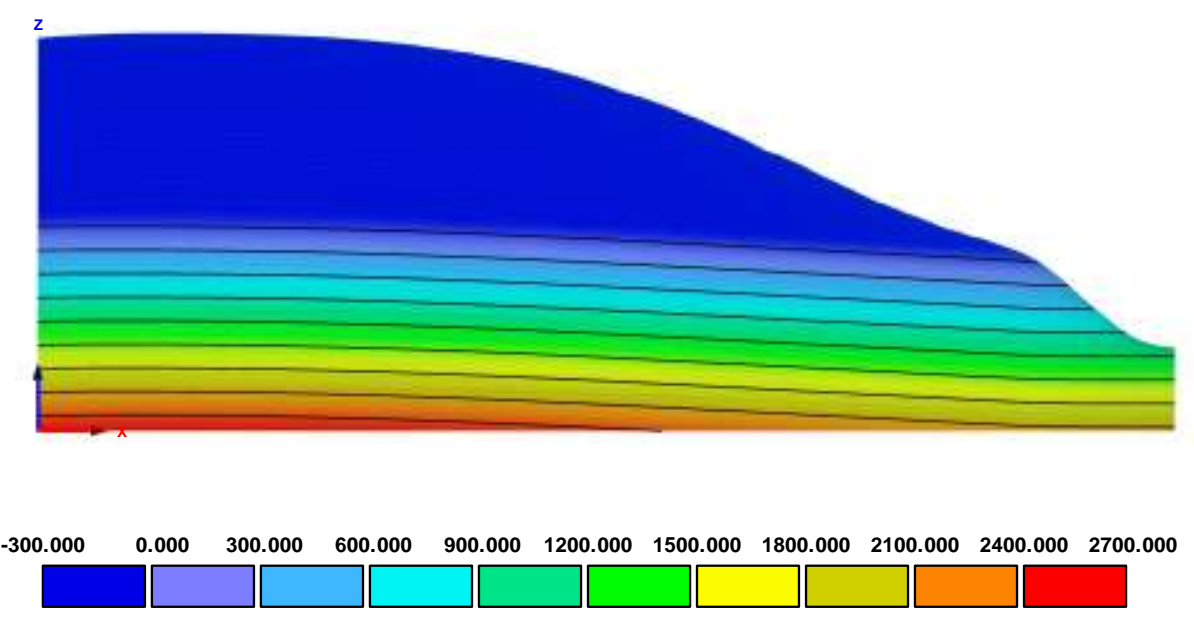

Figura 6 - Perfil de carga de pressão $(\mathrm{cm})$ em regime permanente

Figure 6 - Pressure head profile $(\mathrm{cm})$ subjected to steady steate flow

Adicionalmente, com base nas curvas de retenção de água no solo e características de recarga foi possível estabelecer a distribuição de umidade em regime permanente. A Figura 7 mostra o perfil de umidade em regime permanente. Na Figura 6, a posição da isolinha correspondente à pressão capilar nula é coincidente com a do início do lençol freático da Figura 7. Adicionalmente, a Figura 7 mostra que os teores de umidade nos solos das formações Rio Claro e Corumbataí, abaixo do lençol freático, são coincidentes com a máxima saturação obtida na curva de pressão capilar da Tabela $1\left(\theta_{s}=0,45\right)$. 

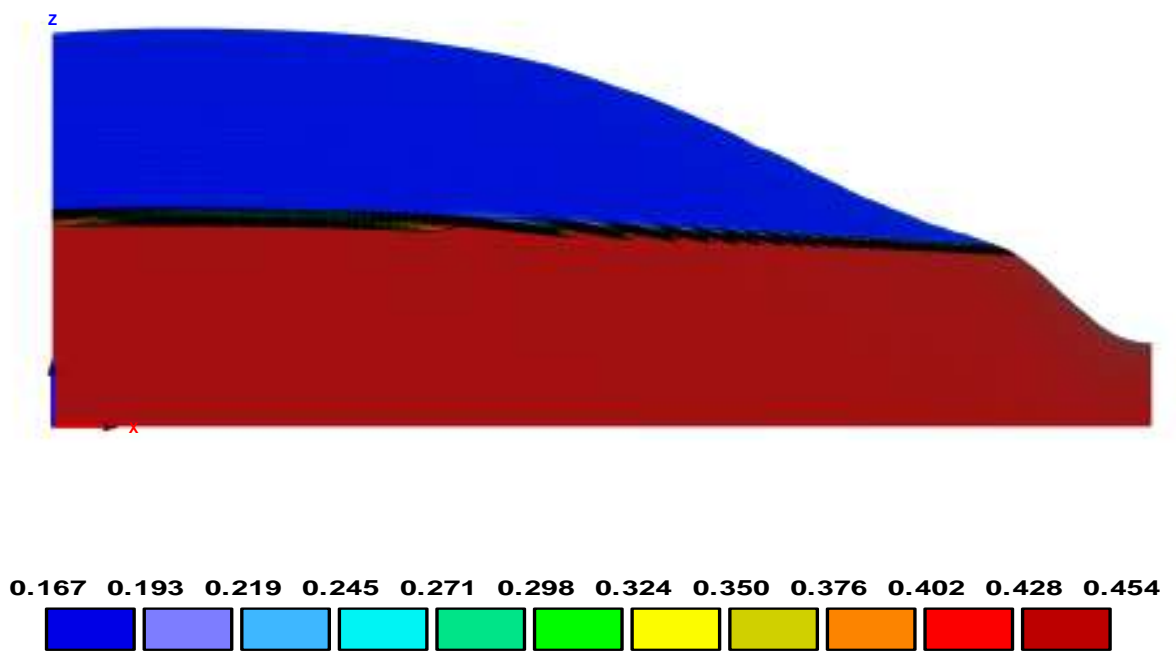

Figura 7 - Perfil de umidade $\left(\mathrm{cm}^{3} / \mathrm{cm}^{3}\right)$ submetido a fluxo em regime permanente Figure 7 - Water content profile $\left(\mathrm{cm}^{3} / \mathrm{cm}^{3}\right)$ subjected to steady state flow

A Figura 8 mostra os resultados da distribuição do soluto da vinhaça com as fontes a 200 metros (F1) e 400 metros (F2) do córrego após 5 anos (Figuras 8a e 8b, respectivamente), 17,5 anos (Figuras $8 \mathrm{c}$ e $8 \mathrm{~d}$, respectivamente) e 35 anos (Figuras 8e e 8f, respectivamente). Na situação mostrada na Figura 8a (fonte $\mathrm{F} 1$, após um período de 5 anos), a dispersão vertical permitiu que o contaminante atingisse o nível freático, devido à maior proximidade de $\mathrm{F} 1$ com o córrego (com NA a aproximadamente $6 \mathrm{~m}$ de profun- didade), em relação à fonte $\mathrm{F} 2$, mais distante e com lençol freático a cerca de $20 \mathrm{~m}$ de profundidade mais distante (Figura 8b). Após um período de 17,5 anos (Figuras 8c e 8d), verifica-se ainda que apenas o soluto proveniente da fonte mais próxima atingiu o córrego. Após 35 anos (Figuras 8e e 8f), os solutos advindos de ambas as fontes atingiram o córrego, porém o deslocamento do soluto da fonte mais próxima (Figuras 8e) se mostrou predominantemente vertical.

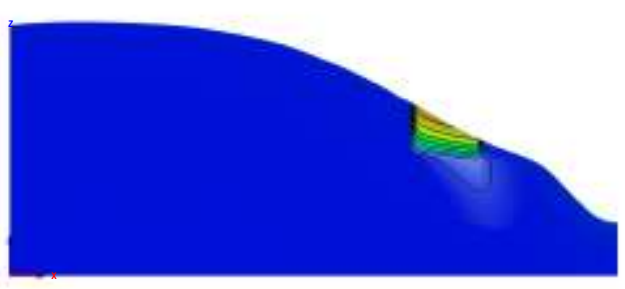

(a)

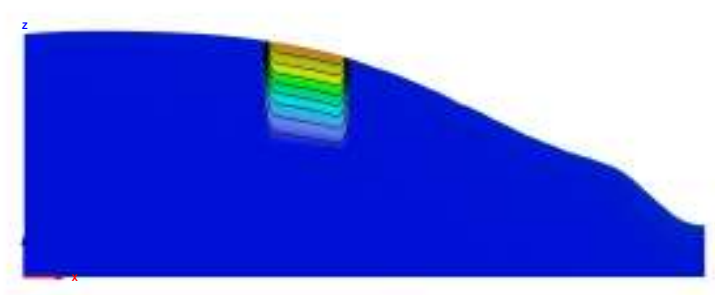

(b) 


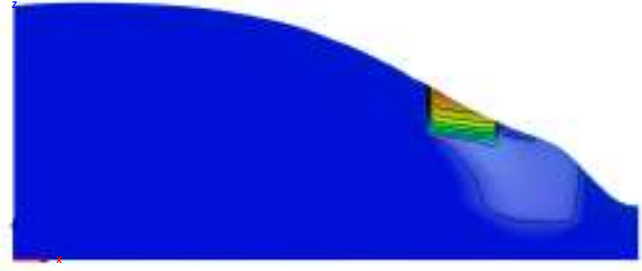

(c)

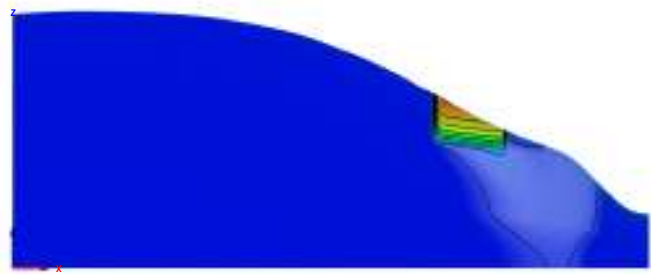

(e)

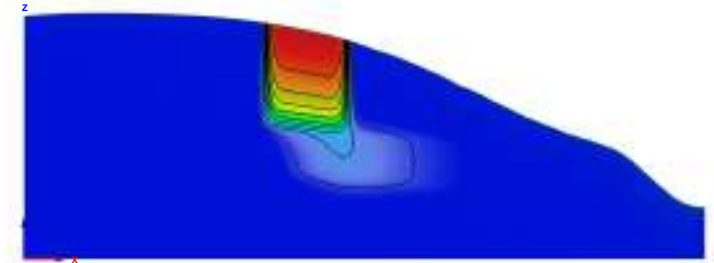

(d)

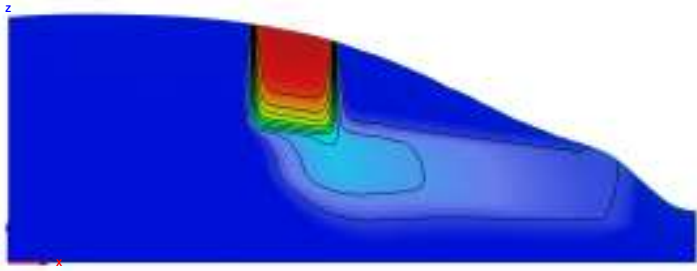

(f)

\section{$\begin{array}{lllllllllll}0.000 & 0.100 & 0.200 & 0.300 & 0.400 & 0.500 & 0.600 & 0.700 & 0.800 & 0.900 & 1.000\end{array}$}

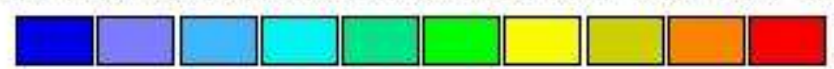

Figura 8 - Distribuição do soluto $\left(\mathrm{mmol} / \mathrm{cm}^{3}\right)$ da vinhaça com distâncias entre as fontes e córrego $\mathrm{F} 1=200 \mathrm{~m}$, $\mathrm{F} 2=400 \mathrm{~m}$ após 5 anos (Figuras $a$ e $b$, respectivamente), 17,5 anos (Figuras $c$ e $d$, respectivamente) e 35 anos (Figuras $e$ e $f$, respectivamente)

Figure 8 - Distribution of solute $\left(\mathrm{mmol} / \mathrm{cm}^{3}\right)$ of vinasse with distances between sources and stream $\mathrm{F} 1=$ 200m, F2 $=400 \mathrm{~m}$ after 5 years (Figures a and b, respectively), 17,5 years (Figures c and d, respectively ) and 35 (Figures e and f, respectively)

Com a finalidade de avaliar a variação da concentração do íon do soluto em relação à chegada na água subterrânea e no córrego foram inseridos pontos de observação na modelagem numérica. Um ponto de observação encontra-se próximo ao contato da zona de descarga (seepage) do córrego e os outros dois pontos de observação, na profundidade do lençol freático logo abaixo do centro de massa de cada fonte.

A Figura 9 mostra o gráfico de concentração $\left(\mathrm{mmol} / \mathrm{cm}^{3}\right)$ do soluto versus tempo (anos) para os pontos de observação localizados abaixo de cada fonte de contaminação (F1 e F2), na profundidade do lençol freático ( $6 \mathrm{~m}$ e $20 \mathrm{~m}$, respectivamente). $\mathrm{O}$ gráfico mostra que a chegada do soluto na água subterrânea (AS) ocorrerá em menos de 1 ano a partir da fonte $\mathrm{F} 1$ e em menos de 3 anos a partir da fonte $\mathrm{F} 2$. A fonte $\mathrm{F} 2$ proveria a máxima concentração na água subterrânea (44\% da concentração inicial), situação que seria atingida após um período de aproximadamente 30 anos. Já a concentração máxima proveniente da fonte $\mathrm{F} 1$ ( $31 \%$ da concentração original) poderia ser atingida em apenas 10 anos. 


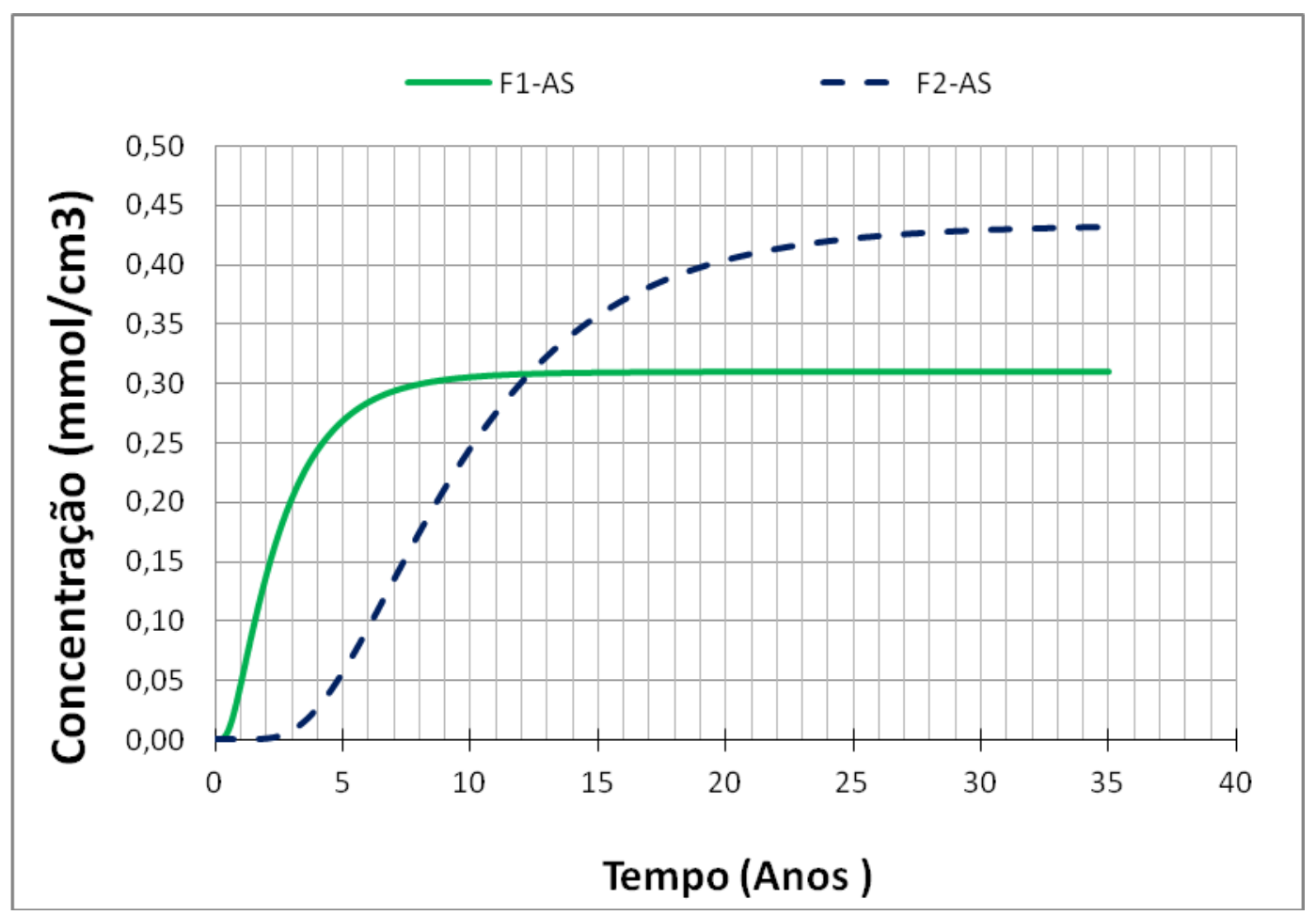

Figura 9 - Concentrações $\left(\mathrm{mmol} / \mathrm{cm}^{3}\right)$ do soluto versus tempo (anos) para os pontos de observação localizados abaixo de cada fonte de contaminação (F1 e F2)

Figure 9 - Concentrations $\left(\mathrm{mmol} / \mathrm{cm}^{3}\right)$ of the solute versus time (years) to the observation points located below each source of contamination (F1 and F2)

A Figura 10 mostra a relação entre as concentrações $\left(\mathrm{mmol} / \mathrm{cm}^{3}\right)$ finais do soluto versus tempo (anos) para o ponto de observação localizado na zona de descarga do córrego. As concentrações finais são provenientes de cada fonte de contaminação (F1 e F2). O gráfico sugere que a chegada do contaminan- te no córrego ocorrerá em apenas 2,2 anos e 14,2 anos a partir das fontes F1 e F2, respectivamente. Adicionalmente, observa-se que, para o período analisado, no máximo $14 \%$ e $10 \%$ da concentração inicial atingirão o córrego após um período de 30 anos e 35 anos a partir das fontes F1 e F2, respectivamente. 


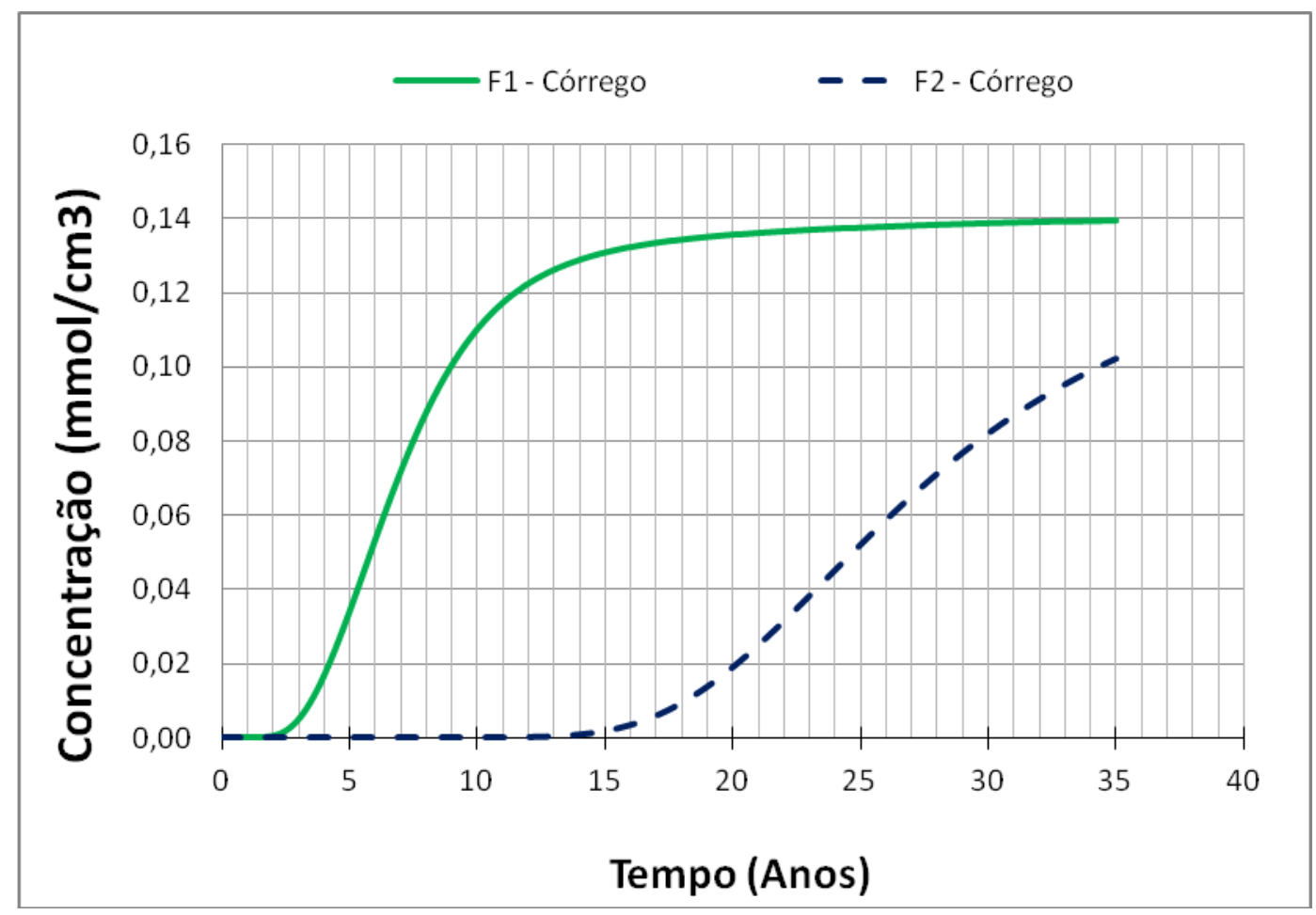

Figura 10 - Concentrações $\left(\mathrm{mmol} / \mathrm{cm}^{3}\right)$ finais do soluto versus tempo (anos) para o ponto de observação localizado na margem do córrego, para as concentrações provenientes de cada fonte de contaminação (F1 e F2)

Figure 10 - Final concentrations $\left(\mathrm{mmol} / \mathrm{cm}^{3}\right)$ of the solute versus time (years) to the observation point on the stream bank, for concentrations from each contamination source (F1 and F2)

\section{CONCLUSÕES}

Foi simulado um modelo conceitual hipotético de contaminação por vinhaça em um perfil de solo típico da cidade de Rio Claro (SP), próximo a áreas de fertirrigação relacionada à atividade sucroalcooleira. A análise numérica das condições simuladas mostra que as águas subterrâneas do local estão sujeitas a contaminação, indicando que o soluto da vinhaça pode alcançar o nível freático em pouco tempo de percolação (1 a 3 anos), dependendo de sua profundidade $(6 \mathrm{~m}$ a 20m). Adicionalmente, concentrações entre $31 \%$ e $44 \%$ da concentração inicial do soluto podem ser atingidas em períodos de tempo de 10 a 30 anos.

Quanto às águas superficiais, também sujeitas à contaminação, o modelo mostra que o córrego Antônio Charadia pode ser atingido em apenas 2,2 anos para uma fonte de contaminação localizada a $200 \mathrm{~m}$ de distância do córrego e nível de água subterrânea (NA) a $6 \mathrm{~m}$ de profundidade ou 14,2 anos pa- ra uma fonte a $400 \mathrm{~m}$ e NA a $20 \mathrm{~m}$ de profundidade. Quanto às concentrações o modelo aponta que um máximo de $14 \%$ da concentração inicial chegará ao córrego após um período de 30 anos.

Cabe ressaltar que as localizações das fontes são hipotéticas, e que modelos numéricos incorporam simplificações de sistemas naturais complexos. No entanto, é importante observar que os períodos de chegada de contaminantes são curtos e as concentrações finais podem comprometer a qualidade das águas superficiais e subterrâneas.

\section{AGRADECIMENTOS}

Os autores agradecem à Fundação de Amparo à Pesquisa do Estado de São Paulo (Processo FAPESP N ${ }^{0}$ 2012/5097-2) pelo financiamento deste projeto. 


\section{REFERÉNCIAS}

ALFARO SOTO, M. A; CHANG, H. K; VILAR, O. M. Avaliação do escalonamento fractal de alguns solos Brasileiros. Revista Brasileira de Geociências v. 38, 253-263, 2008.

ALFARO SOTO, M. A; CHANG, H. K; VILAR, O. M. Permeabilidade relativa em zona vadosa com porosidade bimodal: um estudo em solos brasileiros. Águas Subterrâneas. v. 27, n. 2, 93-103, 2013.

ASTM. Standard Guide for Risk-Based Corrective Action Applied at Petroleum Release Sites. ASTM E1739-95. American Society for Testing and Materials, West Conshohocken, PA, 1995.

BRITTO, F.L; ROLIM, M.M; PEDROSA, E. M.R. Concentração de cátions presentes no lixiviado de solos tratados com vinhaça. Revista Eng. Agrícola Jaboticabal, v. 27, n. 3, p.773-781, 2007.

CARNIER, D; CHANG, H.K. Aplicação do método de flutuação de nível da água para a estimativa de recarga: exemplo do aquífero Rio Claro. Revista Águas Subterrâneas. v. 22, n 1, p.39-48, 2008.

CONAB. Companhia Nacional de Abastecimento. Acompanhamento de safra Brasileira: cana de açúcar, Safra 2014/2015, v. 1, n. 3, 28p, 2014.

DA SILVA, N.F; LELIS J.A; TEIXEIRA, M.B; CUNHA, F.N; MIRANDA, J.H; COELHO, R.D. Distribuição de solutos em coluna, de solo com vinhaça. Irriga Botocatu, Ed. Especial, p: 340-350, 2012.

DURNER, W. Hydraulic conductivity estimation for soils with heterogeneous pore structure, Water Resour. Res., v. 30, 211-223, 1994.

GOOGLE EARTH. http://www.google.com.br/intl/ptBR/earth/. Acesso em dezembro de 2014.

IPT. Instituto de Pesquisas Tecnológicas. Mapa geoformológico do estado de São Paulo. Escala
1:1.000.000. Divisão de Minas e Geologia Aplicada do Instituto de pesquisas tecnológicas do estado de São Paulo. São Paulo. IPT, 1981.

LEVANTAMENTO PEDOLOGICO SEMIDETALHADO DO ESTADO DE SÃO PAULO. Serviço Nacional de Levantamento e Conservação do Solo. Instituto Agronômico. Quadricula II São Carlos, Memorial Descritivo, Campinas $\mathrm{n}^{\circ} 98,188 \mathrm{p}$. Escala1:100.000, 1984.

MARINHO, F. A. M. Medição de sucção com o método do papel filtro. In: X Congresso Brasileiro de Mecânica dos Solos e Engenharia de Fundação, Foz de Iguaçu, Paraná, Anais..., v. 2, p. 515-522, 1994.

MILLINGTON, R. J., AND QUIRK, J.M. Permeability of porous solids. Trans. Faraday Soc. v. 57, p. 1200-1207, 1961.

REYNOLDS, W.D.; ELRICK, D.E.; CLOTHIER, B.E. The constant head well permeameter: Effect of unsaturated flow. Soil Sci., v. 139, p. 173-180, 1985.

ŠIMU゚NEK, J.; VAN GENUCHTEN, M.TH.; ŠEJNA, M. The HYDRUS software package for simulating the two- and three-dimensional movement of water, heat, and multiple solutes in variably-saturated media; Technical manual, Version 2.0, PC-Progress, Prague, Czech Republic, 258 p., 2011.

UYEDA, C.A; DE MIRANDA, J.H;DUARTE S.N; DE MEDEIROS, P.R.F; DIAS, C.T.S. Influence of vinasse application in hydraulic conductivity in three soils. Eng. Agricola Jaboticabal, v.33, No.4, p.689898, 2013.

VAN GENUCHTEN, M.TH. A closed-form equation for predicting the hydraulic conductivity of unsaturated soils. Soil Sci. Soc. Am. J., v. 44, n. 5, p. 892-898, 1980. 